\title{
ON A CLASS OF DISTRIBUTIONS STABLE UNDER RANDOM SUMMATION
}

\author{
L. B. KLEBANOV, * Charles University \\ A. V. KAKOSYAN, Yerevan State University \\ S. T. RACHEV, Stony Brook University \\ G. TEMNOV, ${ }^{* *}$ University College Cork
}

\begin{abstract}
We study a family of distributions that satisfy the stability-under-addition property, provided that the number $v$ of random variables in a sum is also a random variable. We call the corresponding property $v$-stability and investigate the situation when the semigroup generated by the generating function of $v$ is commutative. Using results from the theory of iterations of analytic functions, we describe $v$-stable distributions generated by summations with rational generating functions. A new case in this class of distributions arises when generating functions are linked with Chebyshev polynomials. The analogue of normal distribution corresponds to the hyperbolic secant distribution.
\end{abstract}

Keywords: Stability; random summation; characteristic function; hyperbolic secant distribution

2010 Mathematics Subject Classification: Primary 60E07

Secondary 60E10

\section{Introduction}

In many applications of probability theory certain specific classes of distributions have become very useful, usually called 'fat-tailed' or 'heavy-tailed' distributions. The stable distributions that originate from the central limit problem are probably most popular among the heavy-tailed distributions; however, there is a wide collection of classes of distributions, all related to stable distributions in many various ways, but often these relations are not obvious.

Furthermore, certain generalizations of stable distributions are known, using sums of random numbers of random variables (instead of sums with a deterministic number of summands); see, e.g. [6] and [13] for the examples including the so-called $v$-stable distributions, introduced independently in [1] and [12].

In the present paper we focus on presenting further examples of strictly $v$-stable random variables that could be useful in practical applications, such as in financial mathematics.

\section{Definition of strictly $v$-stable random variables, properties, and examples}

In this section we give a general insight into strictly $v$-stable distributions and provide some examples.

Received 6 January 2011; revision received 15 November 2011.

* Postal address: Department of Probability and Statistics, Charles University, Prague Sokolovska 83, Prague-8, CZ 18675, Czech Republic. Email address: klebanov@chello.cz

** Postal address: School of Mathematical Sciences, University College Cork, Western Gateway Building, Western Road, Cork, Ireland. Email address: g.temnov@ucc.ie 


\subsection{Basic definitions}

Let $X, X_{1}, X_{2}, \ldots, X_{n}, \ldots$ denote a sequence of independent and identically distributed (i.i.d.) random variables, and let $\left\{v_{p}, p \in \Delta\right\}$ be a family of discrete random variables (RVs) taking values in the set of natural numbers $\mathbb{N}$. Assume that this family does not depend on the sequence $\left\{X_{j}, j \geq 1\right\}$ and that, for $\Delta \subset(0,1)$,

$$
\mathrm{E} v_{p}=\frac{1}{p} \text { for all } p \in \Delta .
$$

Definition 1. We say that the RV $X$ has a strictly $\nu$-stable distribution if, for all $p \in \Delta$,

$$
X \stackrel{\mathrm{D}}{=} p^{1 / \alpha} \sum_{i=1}^{v_{p}} X_{j}
$$

where $\alpha \in(0,2]$ is called the index of stability.

After this general definition, a narrower class is defined for $\alpha=\frac{1}{2}$.

Definition 2. We call the RV $X$ a strictly $\nu$-normal $\mathrm{RV}$ if $\mathrm{E} X=0, \mathrm{E} X^{2}=\infty$, and

$$
X \stackrel{\mathrm{D}}{=} p^{1 / 2} \sum_{i=1}^{v_{p}} X_{j} \quad \text { for all } p \in \Delta .
$$

Closely related to the stability property is the property of infinite divisibility, so we also give the following definition.

Definition 3. The RV $X$ has a strictly $\nu$-infinitely divisible distribution if, for any $p \in \Delta$, there exists an $\operatorname{RV} Y^{(p)}$ such that

$$
X \stackrel{\mathrm{D}}{=} \sum_{j=1}^{v_{p}} Y_{j}^{(p)} \quad \text { with } Y^{(p)}, Y_{1}^{(p)}, \ldots, Y_{n}^{(p)}, \ldots \text { i.i.d. RVs. }
$$

A powerful tool for investigating distributions' properties is the generating function $\mathcal{P}_{p}(z):=$ $\mathrm{E}\left[z^{v_{p}}\right]$. Moreover, we denote by $\mathcal{A}$ the semigroup generated by the family $\left\{\mathcal{P}_{p}, p \in \Delta\right\}$, with the operation of functional composition.

\subsection{Summary of the known results}

With regards to the definitions above, the following results are known (see, e.g. [1], [11], and [12] for proofs and details).

Theorem 1. For the family $\left\{\mathcal{P}_{p}, p \in \Delta\right\}$ with $\mathrm{E}\left[v_{p}\right]=1 / p$, there exists a strictly v-normal distribution if and only if the semigroup $\mathcal{A}$ is commutative.

Suppose that we have a commutative semigroup $\mathcal{A}$. Then the following properties are known (see, e.g. [7] for proofs and details).

(P1) The system

$$
\varphi(t)=\mathcal{P}_{p}(\varphi(p t)) \quad \text { for all } p \in \Delta
$$

of functional equations has a solution that satisfies the initial conditions

$$
\varphi(0)=1, \quad \varphi^{\prime}(0)=-1 .
$$


The solution is unique. In addition, there exists a cumulative distribution function (CDF) $A(x)$ (with $A(0)=0$ ) such that

$$
\varphi(t)=\int_{0}^{\infty} \mathrm{e}^{-t x} \mathrm{~d} A(x) .
$$

(P2) The characteristic function (CF) of the strictly $\nu$-normal distribution has the form

$$
f(t)=\varphi\left(a t^{2}\right), \quad a>0 .
$$

(P3) A CF $g(t)$ is a CF of a $v$-infinitely divisible RV if and only if there exists a CF $h(t)$ of an infinitely divisible (in the usual sense) RV such that

$$
f(t)=\varphi(-\ln h(t))
$$

Relation (2) allows one to obtain explicit representations of CFs of strictly $v$-stable distributions. Clearly, they are obtained through applying (2) to the CF $(h(t))$ of strictly stable (in the usual sense) distributions. Moreover, note that the $\mathrm{CF} \varphi($ ait $), a \in \mathbb{R}^{1}$, is the CF of an analogue of the degenerate RV, and that, for the RV with such a CF, the following analogue of the law of large numbers exists.

Theorem 2. Let $X_{1}, X_{2}, \ldots, X_{n}, \ldots$ be a sequence of i.i.d. $R V$ s with finite first moment, and let $\left\{v_{p}, p \in \Delta\right\}$ be a family of $R V$ s taking values in $\mathbb{N}$, independent of the sequence $\left\{X_{j}, j=\right.$ $1,2, \ldots\}$. Assume that $\mathrm{E}\left[v_{p}\right]=1 / p$ and that the semigroup $\mathcal{A}$ is commutative.

Then the series $p \sum_{j=1}^{v_{p}} X_{j}$ is convergent in distribution as $p \rightarrow 0$, and the limit is an $R V$ having the $C F \varphi($ ait $)$.

The proof of this theorem follows straightforwardly from property (P1) and the transfer theorem of Gnedenko (see [6]). For the reader's convenience, we give the formulation of the transfer theorem.

Theorem 3. For any integer $n$, let $\xi_{n 1}, \xi_{n 2}, \ldots$ be a sequence of i.i.d. RVs. Let $k_{n}$ be a sequence of positive integers, and let $v_{n}$ be a sequence of integer-valued RVs. We assume that, for each $n, v_{n}$ and $\xi_{n 1}, \xi_{n 2}, \ldots$ are independent. If

(i) $\mathrm{P}\left\{\sum_{k=1}^{k_{n}} \xi_{n k} \leq x\right\} \rightarrow \Phi(x)$ as $n \rightarrow \infty$; and

(ii) $\mathrm{P}\left\{v_{n} / k_{n} \leq x\right\} \rightarrow A(x)$ as $n \rightarrow \infty$, where $\Phi(x)$ and $A(x)$ are distribution functions, then

(iii) $\mathrm{P}\left\{\sum_{k=1}^{v_{n}} \xi_{n k} \leq x\right\} \rightarrow \Psi(x)$ as $n \rightarrow \infty$.

The distribution $\Psi(x)$ is determined by its characteristic function $\psi$, defined by

$$
\psi(t)=\int_{0}^{\infty}[\phi(t)]^{z} \mathrm{~d} A(z),
$$

where $\phi$ is the CF corresponding to $\Phi$.

In the following subsection we discuss several particular examples of strictly $\nu$-normal and strictly $\nu$-stable distributions. 


\subsection{Examples and the outline of the problem}

Example 1. (The usual stability.) Assume that $v_{p}=1 / p$ with probability 1 , where $p \in \Delta=$ $\left\{1, \frac{1}{2}, \ldots, 1 / n, \ldots\right\}$, and so $\mathcal{P}_{p}(z)=z^{1 / p}$.

Clearly, here the semigroup $\mathcal{A}$ is commutative. Furthermore,

$$
\varphi(t)=\exp \{-t\}=\int_{0}^{\infty} \mathrm{e}^{-t x} \mathrm{~d} A(x),
$$

where $A(x)$ is a CDF with a single unit-sized jump at $x=1$. In this example the strictly $v$-normal CF is the CF of the normal (in the usual sense) RV with the zero mean.

Example 2. (The geometric summation scheme.) Suppose that $v_{p}$ has a geometric distribution:

$$
\mathrm{P}\left\{v_{p}=k\right\}=p(1-p)^{k-1}, \quad k=1,2, \ldots, p \in(0,1) .
$$

Clearly, here $\mathrm{E}\left[v_{p}\right]=1 / p$ and $\mathcal{P}_{p}(z)=p z /(1-(1-p) z), p \in(0,1)$. It is quite straightforward to check that $\mathcal{A}$ is commutative. Moreover, a direct calculation gives $\varphi(t)=$ $1 /(1+t)=\int_{0}^{\infty} \mathrm{e}^{-t x} \mathrm{e}^{-x} \mathrm{~d} x$, i.e. $A(x)$ is the CDF of the exponential distribution. So a $v$-analogue of the strictly normal distribution is the Laplace distribution with the CF $f(t)=1 /\left(1+a t^{2}\right)$.

Example 3. (Branching process scheme.) Let $\mathcal{P}(z)$ be some generating function, with $\mathcal{P}^{\prime}(1)=$ $1 / p_{0}>1$ (so that $p_{0}=1 / \mathcal{P}^{\prime}(1)$, with $p_{0}<1$ ).

Now consider a family given by $\mathcal{P}^{0 n}(z)=\mathscr{P}^{0(n-1)}(\mathcal{P}(z)), n=1,2, \ldots$ Related to this family is another family of the RVs $v_{p}: \mathcal{P}_{p}(z)=\mathscr{P}^{0 n}(z), p \in\left\{1 / p_{0}^{n}, n=1,2, \ldots\right\}=: \Delta$.

Clearly, the semigroup $\mathcal{A}$ coincides with the family $\left\{\mathcal{P}_{p}, p \in \Delta\right\}$. The $\mathrm{CF} \varphi(t)$ is a solution of the functional equation $\varphi(t)=\mathcal{P}\left(\varphi\left(p_{0} t\right)\right)$.

Additional references on this topic are [1], [12], and [14].

As mentioned in the introduction, we aim to widen the collection of examples that involve random summation with the commutative semigroup $\mathcal{A}$. For that purpose, we address the description of pairs of certain commutative generating functions $\mathcal{P}$ and $\mathcal{Q}$, i.e. those for which the balance equality $\mathcal{P} \circ \mathcal{Q}=\mathcal{Q} \circ \mathcal{P}$ holds, but including only the case when there exists no function $\mathscr{H}$ such that $\mathcal{P}=\mathscr{H}^{0 k}$ and $\mathcal{Q}=\mathscr{H}^{0 m}$ for some $k, m \in \mathbb{N}$ (which would be exactly the case of Example 3).

In general, the problem of describing all such commutative pairs of generating functions unfortunately appears very complicated. However, certain special cases are rather straightforward for consideration. In order to address the problem, we will use certain notions typical for the theory of iterations of analytic functions, which we outline in the next section.

\section{Theoretical justification via iterations of analytic functions}

Let $\mathcal{P}$ be a rational function with degree greater than or equal to 2 . Denote by $\mathcal{P}^{0 n}$ its $n$th iteration. The functions $\mathcal{P}$ and $\mathcal{Q}$ are called conjugates if there exists a linear-fractional function $R$ such that $\mathcal{P} \circ R=R \circ \mathcal{Q}$.

A subset $E$ of the extended complex plane $\overline{\mathbb{C}}$ is called completely invariant if its complete inverse image $\mathcal{P}^{-1}(E)$ coincides with $E$. The maximal finite, completely invariant set $E(\mathcal{P})$ exists and is called the exceptional set of the function $\mathcal{P}$. It always holds that $\operatorname{card} E(\mathcal{P}) \leq 2$. Moreover, if $\operatorname{card} E(\mathcal{P})=1$ then the function $\mathcal{P}$ is a conjugate to a polynomial, while, for $\operatorname{card} E(\mathcal{P})=2$, the function $\mathcal{P}$ is a conjugate to $\mathcal{Q}(z)=z^{n}, n \in \mathbb{Z} \backslash\{0,1\}$. Clearly, $E(\mathcal{Q})=$ $\{0, \infty\}$. 
If $\mathcal{P}$ is a rational function then it is known (see, e.g. [4]) that there is a finite number of open sets $F_{i}, i=1, \ldots, r$, which are left invariant by the operator $\mathcal{P}$ and are such that the following conditions hold:

(C1) the union $\bigcup_{i=1}^{r} F_{i}$ is dense on the plane;

(C2) $\mathcal{P}$ behaves regularly on each of $F_{i}$.

Condition (C2) means that the termini of the sequences of iterations generated by the points of $F_{j}$ are either precisely the same set, which is then a finite cycle, or they are finite cycles of finiteor annular-shaped sets that are lying concentrically. In the first case the cycle is attracting and in the second case the cycle is neutral.

The sets $F_{j}$ are the Fatou domains of $\mathcal{P}$, and their union is the Fatou set $F(\mathcal{P})$ of $\mathcal{P}$.

The complement of $F(\mathcal{P})$ is the Julia set $\mathscr{g}(\mathcal{P})$ of $\mathcal{P}$. Note that $\mathscr{g}(\mathcal{P})$ is either nowhere dense and uncountable or $\mathscr{g}(\mathcal{P})=\overline{\mathbb{C}}$. Like $F(\mathscr{P}), \mathscr{g}(\mathcal{P})$ is left invariant by $\mathcal{P}$, and on this set the iteration is repelling, meaning that $|\mathcal{P}(z)-\mathscr{P}(w)|>|z-w|$ for all elements $w$ in a neighborhood of $z$ (within $\mathscr{g}(\mathcal{P})$ ). This means that $\mathcal{P}(z)$ behaves chaotically on the Julia set. Although there are points in the Julia set whose sequence of iterations is finite, there is only a countable number of such points (and they make up an infinitely small part of the Julia set). The sequences generated by points outside this set behave chaotically, a phenomenon called deterministic chaos. Let $z_{0}$ be a repelling fixed point of the function $\mathcal{P}$, and let $\lambda=\mathcal{P}^{\prime}\left(z_{0}\right)$. Define $\Lambda: z \rightarrow \lambda z$. Then there exists a unique solution of the Poincaré equation,

$$
F \circ \Lambda=\mathcal{P} \circ F, \quad F(0)=z_{0}, \quad F^{\prime}(0)=1,
$$

that is meromorphic in $\overline{\mathbb{C}}$.

Now let

$$
\ell(\mathcal{P})=F^{-1}(\mathscr{g}(\mathcal{P})) .
$$

If, for two functions $\mathcal{P}$ and $\mathcal{Q}$, we have $\mathcal{P} \circ \mathcal{Q}=\mathcal{Q} \circ \mathcal{P}$ then they have the same function $F$.

We have the following two possibilities:

1. $\ell(\mathcal{P})=\mathbb{C}$ when $\mathscr{g}(\mathcal{P})=\overline{\mathbb{C}}$;

2. $\ell(\mathcal{P})$ is nowhere dense and consists of analytic curves.

Fatou [5] and Julia [9] investigated the problem of identifying $\mathcal{P}$ and $\mathcal{Q}$ for which $\mathcal{P} \circ \mathcal{Q}=$ $\mathcal{Q} \circ \mathcal{P}$. It turned out that in this case $\mathcal{P}$ and $\mathcal{Q}$ can be reduced by a conjugacy to either the form $\mathcal{P}(z)=z^{m}$ and $\mathcal{Q}(z)=z^{n}$ or the form $\mathcal{P}(z)=T_{m}(z)$ and $\mathcal{Q}(z)=T_{n}(z)$, where $T_{k}$ is the Chebyshev polynomial given by $\cos (k \zeta)=T_{k}(\cos \zeta)$.

\section{Main results}

\subsection{A new example}

Let us return to the study of $v$-normal and $v$-stable RVs. Recall that we consider the family $\left\{v_{p}, p \in \Delta\right\}$ taking its values in $\mathbb{N}=\{1,2, \ldots\}$. As before, we work with the generating function $\mathcal{P}_{p}(z)=\mathrm{E}\left[z^{v_{p}}\right]$ of $v_{p}$. The important result that we stressed says that a strictly $\nu$-normal or strictly $\nu$-stable RV exists if and only if the semigroup $\mathcal{A}$ generated by $\left\{\mathcal{P}_{p}, p \in \Delta\right\}$ is commutative. If $\mathcal{P}_{p}, p \in \Delta$, is a rational function (with degree less than or equal to 2 ) satisfying condition $(\mathrm{C} 2)$ then either $\mathcal{P}_{p}(z)$ is reduced to the form $\widetilde{\mathcal{P}}_{p}(z)=z^{1 / p}, p \in\{1 / n, n=$ $1,2, \ldots\}$, in which case we deal, in fact, with the classical (deterministic) summation scheme, or $\mathcal{P}_{p}(z)$ is reduced to the form $\mathcal{P}_{p}(z)=T_{1 / \sqrt{p}}(z), p \in\left\{1 / n^{2}, n=1,2, \ldots\right\}$. Clearly, the 
polynomial $T_{m}(z)$ is not a generating function itself, but a function to which it is a conjugate, namely,

$$
\mathcal{P}_{p}(z)=\frac{1}{T_{1 / \sqrt{p}}(1 / z)}, \quad p \in\left\{\frac{1}{n^{2}}, n=1,2, \ldots\right\},
$$

which is indeed a generating function (a fact that we prove below). Moreover, below we consider in some detail a family of $\operatorname{RVs}\left\{v_{p}, p \in\{1 / n, n=1,2, \ldots\}\right\}$ that have generating functions of the form (3), and investigate the corresponding strictly $v$-normal and strictly $\nu$-stable distributions.

Lemma 1. Let $P_{n}(x)$ be a polynomial with $\operatorname{deg} P_{n}=n$ in even powers of $x$ whose zeros are all within the interval $(-1,1)$. Let $P_{n}(1)=1$ and the polynomial have positive loading coefficient. Then, for any natural number $k$, the function

$$
\mathcal{P}(x)=\frac{x^{k}}{P_{n}(1 / x)}
$$

is a generating function.

Proof. Represent $P_{n}(x)$ as

$$
P_{n}(x)=b_{0}+b_{1} x+\cdots+b_{n} x^{n}=b_{n} \prod_{j=1}^{n}\left(x-a_{j}\right),
$$

where $a_{j}(j=1, \ldots, n)$ are the zeros of the polynomial $P_{n}$ sorted in order of size. As $P_{n}$ is a polynomial in even powers of $x$, if $a_{j}$ is a zero of $P_{n}$ then $-a_{j}$ is also a zero of $P_{n}$. Therefore,

$$
\begin{aligned}
\frac{1}{P_{n}(1 / x)} & =\frac{1}{b_{n} \prod_{j=1}^{n}\left(1 / x-a_{j}\right)} \\
& =\frac{1}{b_{n} \prod_{j=1}^{n / 2}\left(1 / x-a_{j}\right)\left(1 / x+a_{j}\right)} \\
& =\frac{1}{b_{n}} \prod_{j=1}^{n / 2} \frac{1}{\left(1 / x-a_{j}\right)\left(1 / x+a_{j}\right)} \\
& =\frac{1}{b_{n}} \prod_{j=1}^{n / 2} \frac{x^{2}}{1-a_{j}^{2} x^{2}} .
\end{aligned}
$$

Obviously,

$$
\frac{x^{2}}{1-a_{j}^{2} x^{2}}=\sum_{k=0}^{\infty} a_{j}^{2 k} x^{2 k+2}
$$

is a series with positive (nonnegative) coefficients, converging when $|x| \leq 1$. From (4), it now follows that $\mathcal{P}(x)=x^{k} / P_{n}(1 / x)$ is a series also convergent when $|x| \leq 1$, having nonnegative coefficients, and $\mathcal{P}(1)=1$. Hence, $\mathcal{P}(x)$ is a generating function of some RV.

Corollary 1. Let $T_{n}(x)$ be a Chebyshev polynomial of degree $n$. Then

$$
\mathcal{P}(x)=\frac{1}{P_{n}(1 / x)}
$$

is a generating function of some $R V$ which takes values in $\mathbb{N}$. 
Proof. When $n$ is an even number, the result follows directly from Lemma 1 and the properties of Chebyshev polynomials. For odd $n$, consider the representation $T_{n}(x)=x P_{n-1}(x)$, where $P_{n-1}(x)$ is a polynomial in even powers of $x$, satisfying the conditions of Lemma 1 .

Let us now set $\Delta:=\left\{1 / n^{2}, n=1,2, \ldots\right\}$. Consider the family of generating functions

$$
\mathcal{P}_{p}(z)=\frac{1}{T_{1 / \sqrt{p}}(1 / z)}, \quad p \in \Delta .
$$

Clearly, $\mathcal{P}_{p_{1}} \circ \mathcal{P}_{p_{2}}=\mathcal{P}_{p_{2}} \circ \mathcal{P}_{p_{1}}$ for all $p_{1}, p_{2} \in \Delta$, owing to the well-known property of Chebyshev polynomials stating that $T_{n}\left(T_{m}(x)\right)=T_{n m}(x)$. In other words, the semigroup generated by the family $\left\{\mathcal{P}_{p}, p \in \Delta\right\}$ is commutative. It follows (see, e.g. [11]) that there exists a solution to the system of equations

$$
\varphi(t)=\mathcal{P}_{p}(\varphi(p t)), \quad p \in \Delta,
$$

satisfying the initial conditions

$$
\varphi(0)=1, \quad \varphi^{\prime}(0)=-1,
$$

and the solution is unique.

Since $T_{n}(x)=\cos (n \arccos x)=\cosh (n \operatorname{arccosh} x)$, direct substitution shows that

$$
\varphi(t)=\frac{1}{\cosh (\sqrt{2 t})}
$$

satisfies (5) and (6). Hence, the function

$$
f(t)=\frac{1}{\cosh (a t)}, \quad a>0,
$$

is actually a CF of a strictly $v$-normal RV. The CF (8) is, in fact, well known-it is the CF of the hyperbolic secant distribution. Clearly, $a$ here is the scale parameter. When $a=1, \mathrm{CF}(8)$ is the standard hyperbolic secant distribution, whose probability density function is of the form

$$
p(x)=\frac{1}{2} \operatorname{sech}\left(\frac{\pi x}{2}\right),
$$

while the $\mathrm{CDF}$ is

$$
F(x)=\frac{2}{\pi} \arctan \left[\exp \left(\frac{\pi x}{2}\right)\right] .
$$

Furthermore, in order to obtain the expression for the CF of strictly $v$-stable distributions, we simply need to apply relation (2) to the strictly stable (in the usual sense) CF $h$.

\subsection{An interesting property}

The function $\varphi$ in (7) is interesting in its own right, and we will address its properties and consider its CDF $A(x)$ (which corresponds to $\varphi(t)$ via (1)).

Let $W_{1}(t)$ and $W_{2}(t), t \geq 0$, be two independent Wiener processes. Consider the RV

$$
\xi=\int_{0}^{1} W_{1}^{2}(t) \mathrm{d} t+\int_{0}^{1} W_{2}^{2}(t) \mathrm{d} t .
$$


This RV is well studied, and it is known that its Laplace transform equals

$$
\mathrm{E}\left[\mathrm{e}^{-t \xi}\right]=\frac{1}{\cosh (\sqrt{2 t})}
$$

which coincides with $\varphi(t)$ as given in (7). Hence, $A(x)$ is the CDF of the RV $\xi$. On the other hand, as follows from Gnedenko's transfer theorem,

$$
A(x)=\lim _{p \rightarrow 0} \mathrm{P}\left\{p v_{p}<x\right\} .
$$

Theorem 4. Let $\left\{v_{p}<x, p \in \Delta\right\}$ be a family of RVs having generating functions

$$
\mathcal{P}_{p}(z)=\frac{1}{T_{1 / \sqrt{p}}(1 / z)}, \quad p \in \Delta=\left\{\frac{1}{n^{2}}, n=1,2, \ldots\right\} .
$$

Then

$$
\lim _{p \rightarrow 0} \mathrm{P}\left\{p v_{p}<x\right\}=\mathrm{P}\{\xi<x\}
$$

with $\xi$ as in (9).

Theorem 4 may be reformulated in the following way.

Theorem 5. (Theorem 4 reformulated.) Let

$$
\frac{1}{T_{n}(1 / z)}=\sum_{k=0}^{\infty} p_{k}(n) z^{k}
$$

Then

$$
\lim _{n \rightarrow \infty} \sum_{k=0}^{\left[n^{2} x\right]} p_{k}(n)=\mathrm{P}\{\xi<x\}
$$

In Figure 1 we plot $\sum_{k=0}^{\left[n^{2} x\right]} p_{k}(n)$ as a function of $n, n=2, \ldots, 50$. We see that the function attains the constant level rather quickly, and so we can use the asymptotic result for $n>25$.

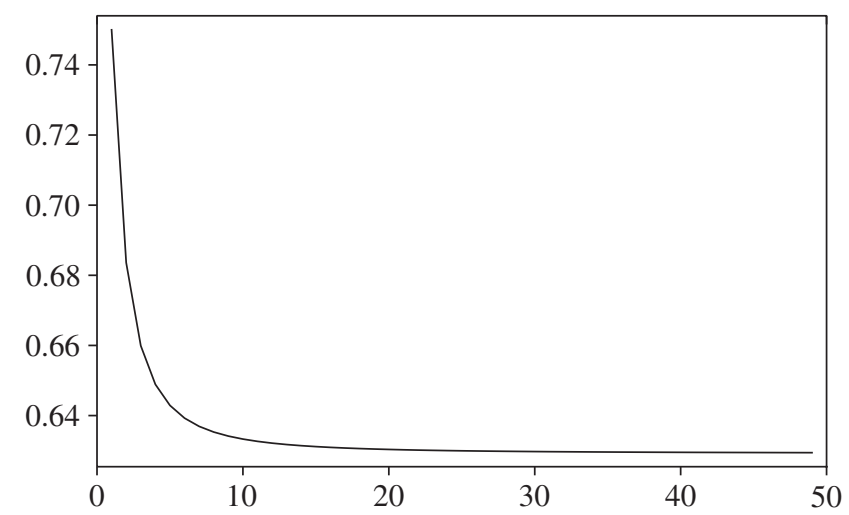

Figure 1: Plot of the $\sum_{k=0}^{\left[n^{2} x\right]} p_{k}(n)$ as the function of $n, n=2, \ldots, 50$. 
Corollary 2. Let $X$ be an $R V$ having the standard hyperbolic secant distribution. Then its distribution can be represented as a scale mixture of normal distributions with zero mean and standard deviation $\sqrt{\xi}$, with $\xi$ as in (9).

To prove Corollary 2, we simply need to write the CF of $X$ in the form $\int_{0}^{\infty} \mathrm{e}^{-t^{2} x} \mathrm{~d} A(x)$, and note that $\mathrm{e}^{-t^{2} x}$ is actually the $\mathrm{CF}$ of the standard normal $\operatorname{RV} N\left(0, \sigma^{2}\right)\left(\sigma^{2}=x\right)$, while $A(x)$ is the $\mathrm{CDF}$ of $\xi$.

Note that there is a certain analogy between the representation $A(x)$ as the CDF of the RV $\xi$ from (9) and the corresponding result in the scheme of the random summation with geometric distribution. Specifically, considering the family $\left\{v_{p}, p \in(0,1)\right\}$ of geometric distribution $\mathrm{P}\left\{v_{p}=k\right\}=p(1-p)^{k-1}, k=1,2, \ldots$, the function $\varphi$ turns into

$$
\varphi(t)=\frac{1}{1+t}=\int_{0}^{\infty} \mathrm{e}^{-t x} \mathrm{~d} A_{1}(x),
$$

with $A_{1}(x)$ the exponential distribution, $A_{1}(x)=1-\mathrm{e}^{-x}$ for $x>0$ and $A_{1}=0$ for $x \leq 0$. It can be checked that if $\eta_{1}$ and $\eta_{2}$ are two independent standard normal RVs, then $A_{1}$ is a CDF of the $\mathrm{RV} \xi_{1}=\eta_{1}^{2}+\eta_{2}^{2}$, which is, in a way, related to (9).

\subsection{Characterizations}

Let us now turn to the characterizations of the distribution of the RV (9) and of the hyperbolic secant distribution.

Theorem 6. Let $X_{1}, \ldots, X_{n}, \ldots$ be a sequence of nonnegative i.i.d. RVs, and let $v_{p}, p \in$ $\left\{1 / n^{2}, n=2, \ldots\right\}$, be a family of $R V s$ with generating function $\mathcal{P}_{p}(z)=1 / T_{1 / \sqrt{p}}(1 / z)$, independent of the sequence $\left\{X_{j}, j \geq 1\right\}$.

If, for some fixed $p \in \Delta$,

$$
X_{1} \stackrel{\mathrm{D}}{=} p \sum_{j=1}^{v_{p}} X_{j},
$$

then $X_{1}$ has the distribution with Laplace transform

$$
\mathrm{E}^{-t X}=\frac{1}{\cosh (\sqrt{a t})}, \quad a>0
$$

Proof. Equality (10), in terms of the Laplace transform $\Psi(t)=\mathrm{E}^{-t X}$, can be represented as

$$
\Psi(t)=\mathcal{P}_{p}(\Psi(p t))
$$

Clearly, the function

$$
\Psi_{a}(t)=\frac{1}{\cosh (\sqrt{a t})}
$$

satisfies (11) for any $a>0$ and, moreover, it is analytic in the strip $|t|<r(r>0)$.

In the following, we use the results of Kakosyan et al. [10]. Example 1.3.2 of [10] shows that $\left\{\Psi_{a}, a>0\right\}$ forms a strongly $\mathcal{E}$-positive family on the set $\mathcal{C}$ of restrictions of Laplace transforms of probability distributions given in $\mathbb{R}_{+}$on an interval $[0, T](0<T<r)$.

Clearly, the operator $A: f \rightarrow \mathcal{P}_{p}(f(p t))$ on $\mathcal{C}$ is intensively monotone.

The statement of Theorem 6 now follows from Theorem 1.1.1 of [10, p. 2]. 
Theorem 7. Let $X_{1}, \ldots, X_{n}, \ldots$ be a sequence of nonnegative i.i.d. RVs, having a symmetric distribution, with $\left\{v_{p}, p \in \Delta\right\}$ as in Theorem 6.

If, for some fixed $p \in \Delta$,

$$
X_{1} \stackrel{\mathrm{D}}{=} p^{1 / 2} \sum_{j=1}^{v_{p}} X_{j},
$$

then $X_{1}$ has the hyperbolic secant distribution with $C F$

$$
f(t)=\frac{1}{\cosh (a t)}, \quad a>0 .
$$

Proof. The proof follows analogously to the proof of Theorem 6, with the difference that instead of Example 1.3.2, we use Example 1.3.1 of [10].

\section{On other random sums of a random number of summands with rational generating functions}

For the case described in Section 3, if two functions $\mathcal{P}$ and $\mathcal{Q}$ satisfy $\mathcal{P} \circ \mathcal{Q}=\mathcal{Q} \circ \mathcal{P}$, then $\mathcal{P}$ and $\mathcal{Q}$ can be reduced by a conjugacy to either the form $\mathcal{P}(z)=z^{m}$ and $\mathcal{Q}(z)=z^{m}$ or the form $\mathcal{P}(z)=T_{m}(z)$ and $\mathcal{Q}(z)=T_{m}(z)$. Therefore, letting $R$ be a fraction-linear function and setting $\mathcal{P}=R^{-1} \circ S \circ R$, where $S$ is either $z^{m}(m>1)$ or $T_{m}(z)$, the following question arises.

- Is there a function $R(z) \neq a * z$ for which $\mathcal{P}$ is a generating function?

In Sections 5.1 and 5.2 we will respectively show that the answer to this question is no in the $S(z)=z^{m}$ case and yes in the $S(z)=T_{n}(m)$ case.

\subsection{Case $S(z)=z^{m}$}

Consider the linear-fractional function

$$
R(z)=\frac{a z+b}{c z+d}, \quad c \neq 0 .
$$

Since $\mathcal{P}=R^{-1} \circ S \circ R$, we have

$$
\mathcal{P}_{m}(z)=P(z)=\frac{d(a z+b)^{m}-b(c z+d)^{m}}{a(c z+d)^{m}-c(a z+b)^{m}} .
$$

However, $\mathcal{P}$ has to be a generating function of an integer-valued random variable $v \geq 1$, and, therefore, we must have

$$
\mathcal{P}_{m}(1)=1, \quad \mathcal{P}_{m}(0)=0,
$$

i.e.

$$
d b^{m}=b d^{m}, \quad(a+b)^{m}(c+d)=(a+b)(c+d)^{m} .
$$

System (12) leads to six subcases:

$$
\begin{array}{rlrl}
a+b & =0, & d=0, \\
a+b & =0, & b=d, \\
c+d=0, & b=0, \\
c+d=0, & b=d, \\
a+b=c+d, & b=0, \\
a+b=c+d, & d=0 .
\end{array}
$$


All the subcases have to be considered separately, but as the method of consideration is similar for all, we consider only one here, namely (17).

In case (17) the generating function $\mathcal{P}_{m}$ has the form

$$
\mathcal{P}_{m}(z)=\frac{d(c+d)^{m} z^{m}}{(c+d)(c z+d)^{m}-c(c+d)^{m} z^{m}} .
$$

We suppose that $c d(c+d) \neq 0$. Defining $p_{1}=c /(c+d)$, rewrite (19), as

$$
\mathcal{P}_{m}(z)=\frac{q_{1} z^{m}}{\left(p_{1} z+q_{1}\right)^{m}-p_{1} z^{m}},
$$

where $q_{1}=1-p_{1}$. It is clear that $\mathcal{P}$ is a generating function if and only if

$$
\mathcal{Q}_{m}(z)=\frac{q_{1}}{\left(p_{1} z+q_{1}\right)^{m}-p_{1} z^{m}}
$$

is also a generating function. However,

$$
\mathcal{Q}_{m}(z)=\frac{q_{1}}{p_{1}^{m}-p_{1}} \frac{1}{\prod_{k=1}^{m}\left(z-z_{k}\right)},
$$

where $z_{k}(k=1,2, \ldots, m)$ are the zeros of the polynomial $\left(p_{1} z+q_{1}\right)^{m}-p_{1} z^{m}$. It is easy to find these zeros. We consider two cases.

Case (i): $p_{1}>0$. In this case the zeros of the polynomial $\left(p_{1} z+q_{1}\right)^{m}-p_{1} z^{m}$ have the form

$$
z_{k}=\frac{q_{1}}{p_{1}^{1 / m} \varepsilon_{m}^{(k)}-p_{1}}, \quad k=1,2, \ldots, m,
$$

where the $\varepsilon_{m}^{(k)}(k=1,2, \ldots, m)$ are the $m$ th roots of unity. In other words,

$$
\varepsilon_{m}^{(k)}=\cos \frac{2(k-1) \pi}{m}+\mathrm{i} \sin \frac{2(k-1) \pi}{m}, \quad k=1,2, \ldots, m .
$$

Using partial fraction decomposition, we write the function $\mathcal{Q}_{m}$ in the form

$$
Q_{m}(z)=\frac{q_{1}}{p_{1}^{m}-p_{1}} \sum_{k=1}^{m} \frac{A_{k}}{z-z_{k}}
$$

where $A_{k}=1 / \prod_{j \neq k}\left(z_{k}-z_{j}\right)$.

Now it is easy to find $\mathcal{Q}_{m}(z)$ as a power series:

$$
\mathcal{Q}_{m}(z)=\frac{q}{p_{1}-p_{1}^{m}} \sum_{s=0}^{\infty}\left(\sum_{k=1}^{m} \frac{1}{\prod_{j \neq k}\left(z_{k}-z_{j}\right)} \frac{1}{z_{k}^{s+1}}\right) z^{s} .
$$

Because $\mathcal{Q}_{m}$ is a generating function, the series in (20) must converge for all complex $z$ with $|z| \leq 1$, the singular point of the series nearest to 0 has to lie on positive semiaxes, and the coefficients of the series have to be nonnegative. Moreover, because $\mathcal{Q}_{m} \circ Q_{m}=\mathcal{Q}_{m^{2}}$, the same properties have to hold not only for one fixed $m$, but for the sequence $m^{l}, l=1,2, \ldots$, i.e. for all functions $\mathcal{Q}_{m}, l=1,2, \ldots$. 
The property of the convergence of the series inside the closed unit circle implies that $\left|z_{k}\right|>1$ for all $k=1,2, \ldots, m$, i.e. we must have

$$
\cos ^{2} \frac{2 \pi n}{m}+p_{1}^{1+1 / m} \cos \frac{2 \pi n}{m} \geq 2 p_{1}+p_{1}^{2 / m} \cos ^{2} \frac{2 \pi n}{m}, \quad n=0,1, \ldots, m-1 .
$$

The last inequality has to hold not only for one fixed value of $m$, but for a sequence $m_{l} \rightarrow \infty$ as $l \rightarrow \infty$ (e.g. for $m_{l}=m^{l}$ ). Passing to the limit as $m \rightarrow \infty$ in the case of $n=0$ we obtain

$$
1 \geq 1+p_{1}
$$

a contradiction. So, in case (i) the function $\mathcal{P}_{m}$ cannot be a generating function.

Case (ii): $p_{1}<0$. Define $p_{2}=-p_{1}>0$ and $q_{1}=1-p_{1}=1+p_{2}$. The polynomial $\left(p_{1} z+q_{1}\right)^{m}-p_{1} z^{m}=\left(-p_{2} z+\left(1+p_{2}\right)\right)^{m}+p_{2} z^{m}$ has the form

$$
z_{k}=\frac{1+p_{2}}{p_{2}^{1 / m} \delta_{m}^{(k)}+p_{2}}, \quad k=1,2, \ldots, m,
$$

where

$$
\delta_{m}^{(k)}=\cos \frac{(2 k-1) \pi}{m}+\mathrm{i} \sin \frac{(2 k-1) \pi}{m}, \quad k=1,2, \ldots, m .
$$

It is easy to calculate that

$$
z_{k}=\frac{\left(1+p_{2}\right)\left(p_{2}^{1 / m} \cos ((2 k-1) \pi / m)+p_{1}-\mathrm{i} \sin ((2 k-1) \pi / m)\right)}{p_{2}^{2 / m}+2 p_{2}^{1+1 / m} \cos ((2 k-1) \pi / m)+p_{2}^{2}}, \quad k=1,2, \ldots, m .
$$

Therefore,

$$
\left|z_{k}\right|=\left(1+p_{2}\right) \frac{1}{\sqrt{p^{2 / m}+2 p_{2}^{1+1 / m} \cos ((2 k-1) \pi / m)+p_{2}^{2}}}, \quad k=1,2, \ldots, m .
$$

If the function $\mathcal{Q}_{m}$ were a generating function then $\left|z_{k}\right|$ with minimal absolute value would lie on the positive semiaxis, but, as (21) shows, it does not. Therefore, in case (ii) the function $\mathcal{P}_{m}$ cannot be a generating function either.

To summarize, $\mathcal{P}_{m}$ cannot be a generating function in case (17). Cases (13)-(16) and (18) are considered similarly.

In conclusion, there are no generating functions conjugate to a power $z^{m}$ or equal to this power.

\subsection{Case $S=T_{m}$}

For any $a \in\left[\frac{1}{2}, 1\right]$ and any integer $m>1$, let us define

$$
\mathcal{P}_{m}(z):=\frac{1}{a T_{m}(1 /(a z)-(1-a) / a)+(1-a)} .
$$

Hypothesis 1. For any $a \in\left(\frac{1}{2}, 1\right]$ and any integer $m>1$, the function $\mathcal{P}_{m}(z)$ defined in (22) is a generating function.

Unfortunately, we cannot prove this hypothesis in full. However, we will give the proof for the case of even $m$. 
Theorem 8. For any $a \in\left(\frac{1}{2}, 1\right]$ and any even integer $m>1$, the function $\mathcal{P}_{m}(z)$ defined in (22) is a generating function.

Proof. Consider the equation $a T_{m}(x)+(1-a)=0$. Its roots are

$$
x_{k}=\cos \left(\frac{1}{m} \arccos \frac{a-1}{a}+\frac{2 \pi k}{m}\right), \quad k=0,1, \ldots, m-1,
$$

and $\left|x_{k}\right|<1$. As $m$ is an even number, the roots are symmetric around 0 . Therefore,

$$
a T_{m}(x)+(1-a)=a 2^{m-1} \prod_{k=0}^{m-1}\left(x-x_{k}\right)=a 2^{m-1} \prod\left(x^{2}-x_{k}^{2}\right),
$$

where the latter product is taken over such $k$ for which $x_{k}>0$. Now we see that

$$
\mathcal{P}_{m}(z)=\left(\frac{a z}{1-(1-a) z}\right)^{m} \frac{1}{\lambda \prod\left(1-x_{k}^{2}(a z /(1-(1-a) z))^{2}\right)},
$$

where $\lambda=a 2^{m-1}$. The statement now follows from the fact that

$$
\frac{1}{1-x_{k}^{2}(a z /(1-(1-a) z))^{2}}=\sum_{j=0}^{\infty}\left(x_{k}\right)^{2 j}\left(\frac{a z}{1-(1-a) z}\right)^{2 j},
$$

and $a z /(1-(1-a) z)$ is the sum of a geometric progression with denominator $1-a$.

The family of functions (22) for any fixed $a \in\left[\frac{1}{2}, 1\right]$ is commutative with respect to convolution, i.e.

$$
\mathcal{P}_{m}\left(\mathcal{P}_{n}(z)\right)=\mathcal{P}_{n}\left(\mathcal{P}_{m}(z)\right)=\mathcal{P}_{m n}(z),
$$

and, consequently, there exists a $\nu$-Gaussian distribution, where the family $\left\{v_{p}, p \in \Delta\right\}$ is defined by the family of corresponding generating functions $\mathcal{P}_{m}(z), m=2,4,6, \ldots$, and the parameter $p$ is defined by $m$ through the relation

$$
p=p(m)=\frac{1}{\mathcal{P}_{m}^{\prime}(1)} .
$$

We will not study the corresponding characteristic functions here. This can be done as above.

\section{Examples with nonrational generating functions}

There exist examples of pairs of commutative functions which are not rational. In this section we provide two classes of such functions: the first was investigated by Melamed [15] and the second appears for the first time here.

Example 4. (See [15] for details.) Consider the family of generating functions

$$
\mathcal{P}_{p}(z)=\frac{p^{1 / m} z}{\left(1-(1-p) z^{m}\right)^{1 / m}},
$$

where $p \in(0,1)$ and $m$ is a fixed positive integer. Obviously, in the case $m=1, \mathcal{P}_{p}(z)$ reduces to the generating function of the geometric distribution, as above. Hence, assume that $m \geq 2$. In that case, it is easy to check that

$$
\varphi(t)=\frac{1}{(1+m t)^{1 / m}},
$$


and, therefore, the CF of the strictly $v$-normal distribution (for the family $\left\{v_{p}, p \in \Delta\right\}$ having generating function (23)) has the form

$$
f(t)=\frac{1}{\left(1+m a t^{2}\right)^{1 / m}},
$$

with parameter $a>0$.

Example 5. Consider the family of functions

$$
\mathcal{P}_{p}(z)=\frac{1}{\left(T_{1 / \sqrt{p}}\left(1 / z^{m}\right)\right)^{1 / m}},
$$

where $p \in\left\{1 / n^{2}, n=2, \ldots\right\}$ and $m \geq 1$ (an integer).

Using a slightly modified version of the proof of Lemma 1 , it is easy to check that $\mathcal{P}_{p}(z)$ is a generating function of some $\mathrm{RV} v_{p}, p \in\left\{1 / n^{2}, n=2, \ldots\right\}$, for any fixed whole number $m \geq 1$ (both $\mathcal{P}_{p}$ and $v_{p}$ both depend on $m$, but we omit this dependence in the notation).

The case $m=1$ has already been considered above. For $m \geq 2$, analogous methods are applicable, and so we will only refer to the results. Specifically,

$$
\varphi(t)=\frac{1}{(\cosh \sqrt{2 m t})^{1 / m}},
$$

while the $\mathrm{CF}$ of the corresponding strictly $v$-normal distribution has the form

$$
f(t)=\frac{1}{(\cosh a t)^{1 / m}},
$$

where $a>0$.

Note that in the case $m=2$, we have the following expressions for the distributions, whose Laplace transforms are given in (24) and (25).

For $m=2$, (24) gives

$$
\varphi(t)=\frac{1}{\sqrt{1+2 t}} .
$$

This function is the Laplace transform of the distribution of the RV $X^{2}$, with $X$ being the standard normal RV.

In a similar way, (25) gives, for $m=2$,

$$
\varphi(t)=\frac{1}{\sqrt{\cosh \sqrt{4 t}}} .
$$

This function is the Laplace transform of the distribution of the RV $I=\int_{0}^{1} X^{2}(t) \mathrm{d} t$, with $X(t)$ the standard Wiener process.

\section{On some applications of $v$-stable distributions}

Let us note that $v$-stable distributions have been used a lot in the modeling of dielectric relaxation in polymers. These distributions were founded as empirical approximations for corresponding relaxation processes. We consider the so-called Cole-Cole relaxation model [2], [3]. The Cole-Cole model is given by the equation

$$
f(\omega)=\frac{1}{1+(\tau \mathrm{i} \omega)^{\alpha}},
$$

where $f$ is the complex dielectric constant, $\omega$ is the angular frequency, and $\tau$ is a time constant. 
The exponent parameter $\alpha$, which takes a value between 0 and 1 , allows us to describe different spectral shapes. When $\alpha=0$, the Cole-Cole model reduces to the Debye model. When $\alpha>0$, the relaxation is stretched, i.e. it extends over a wider range on a logarithmic $\omega$ scale than the Debye relaxation. Clearly, the function $f(\omega)$ is the characteristic function of a geometric-stable RV. This fact supports the assumption that non-Debye relaxation should be connected to the random structure of dielectrics.

Cole-Cole relaxation constitutes a special case of the Havriliak-Negami model (see [8]). In this model

$$
f(\omega)=\frac{1}{\left(1+(\tau \mathrm{i} \omega)^{\alpha}\right)^{\beta}} .
$$

For the case $\beta=1 / m$ with integer $m$, the function $f$ is the characteristic function of the $\nu$-stable distribution from Example 4. This fact shows, however, that, for the Havriliak-Negami model, the character of randomness in dielectrics is slightly different from that of the Cole-Cole case.

It seems natural to suppose that other $v$-stable distributions mentioned in this paper could be applied to the modeling of dielectric relaxation processes for other types of dielectrics.

\section{Random summation and branching processes: alternative approaches to the problem}

This paper and the previous work of the authors place emphasis on $X$, the stable-like RV, and look for those $v$ s for which $X$ is $\nu$-stable. There may be more than one $v$, but there exists a uniqueness theorem that there is at most one $v$ for each fixed value of $E[v]$, a fact also obtained in [14].

The techniques of [14] are based on the use of branching processes. That is, Mallows and Shepp [14] placed emphasis on $v$, and looked for the class of RVs which are $v$-stable. In a sense these two points of view-focusing on $X$ and placing emphasis on $\nu$-are equivalent. The use of branching processes is quite explicit in the earlier work of the present authors.

\section{Acknowledgements}

We would like to thank two anonymous referees for their remarks, comments, and useful suggestions which allowed us to significantly improve the paper, in particular with regards to the analysis of the results in relation to previous works in this field, and with regards to language, style, and presentation of the material in general.

The help of the editors throughout the submission process is also much appreciated.

This work was partially supported by research grant P203/12/0665 (L. Klebanov) and by the SFI research grant 07/MI/008 associated with the Edgeworth Centre for Financial Mathematics (G. Temnov).

\section{References}

[1] Bunge, J. (1996). Composition semigroups and random stability. Ann. Prob. 24, 1476-1489.

[2] Cole, K. S. AND Cole, R. H. (1941). Dispersion and absorption in dielectrics I. Alternating current characteristics. J. Chem. Phys. 9, 341-351.

[3] Cole, K. S. And Cole, R. H. (1942). Dispersion and absorption in dielectrics II. Direct current characteristics. J. Chem. Phys. 10, 98-105.

[4] ErËmenko, A. È. (1989). Some functional equations connected with the iteration of rational functions. Algebra $i$ Analiz 1, 102-116 (in Russian). English translation: Leningrad Math. J. 1 (1990), 905-919.

[5] Fatou, P. (1923). Sur l'itération analytique et les substitutions permutables. J. Math. 2, 343-362.

[6] Gnedenko, B. V. (1983). On some stability theorems. In Stability Problems for Stochastic Models (Moscow, 1982; Lecture Notes Math. 982), Springer, Berlin, pp. 24-31. 
[7] Gnedenko, B. V. And Korolev, V. Y. (1996). Random Summation: Limit Theorems and Applications. CRC Press, Boca Raton, FL.

[8] Havriliak, S. and Negami, S. (1967). A complex plane representation of dielectric and mechanical relaxation processes in some polymers. Polymer 8, 161-210.

[9] Julia, G. (1922). Mémoire sur la permutabilité des fractions rationnelles. Ann. Sci. École Norm. Sup. 39, 131-215.

[10] Kakosyan, A. V., Klebanov, L. B. and Melamed, J. A. (1984). Characterization of Distributions by the Method of Intensively Monotone Operators (Lecture Notes Math 1088), Springer, Berlin.

[11] Klebanov, L. B. (2003). Heavy Tailed Distributions. Matfyz-press, Prague.

[12] Klebanov, L. B. And Rachev, S. T. (1996). Sums of a random number of random variables and their approximations with $v$-accompanying infinitely divisible laws. Serdica Math. J. 22, 471-496.

[13] Klebanov, L. B., Maniya, G. M. and Melamed, I. A. (1984). A problem of Zolotarev and analogs of infinitely divisible and stable distributions in a scheme for summing a random number of random variables. Theory Prob. Appl. 29, 791-794.

[14] Mallows, C. and Shepr, L. (2005). B-stability. J. Appl. Prob. 42, 581-586.

[15] Melamed, J. A. (1989). Limit theorems in the set-up of summation of a random number of independent and identically distributed random variables. In Stability Problems for Stochastic Models (Sukhumi, 1987; Lecture Notes Math. 1412), Springer, Berlin, pp. 194-228. 